\title{
Eficiência agronômica de estirpes de rizóbio inoculadas em feijão- caupi no Cerrado, Gurupi-TO ${ }^{1}$
}

\author{
Agronomic efficiency of rhizobium strains inoculated in cowpea in the Cerrado, \\ Gurupi-TO
}

\author{
Aloísio Freitas Chagas Junior ${ }^{2 *}$, Wagner Rahmeier ${ }^{3}$, Rodrigo Ribeiro Fidelis ${ }^{2}$, Gil Rodrigues dos Santos ${ }^{2}$ e \\ Lillian França Borges Chagas ${ }^{2}$
}

\begin{abstract}
Resumo - A interação do feijão-caupi com bactérias fixadoras de $\mathrm{N}$ atmosférico pode aumentar a produtividade e diminuir os custos de produção. No presente trabalho, avaliou-se, no campo, a eficiência agronômica, através do acúmulo de biomassa da parte aérea, radicular, número e biomassa dos nódulos, eficiência relativa e produtividade de três cultivares de feijãocaupi (BRS Nova Era, BRS Pujante e Vinagre), em experimentos independentes, inoculadas com cinco estirpes de rizóbio, BR 3302 (UFLA 3-84), BR 3301 (INPA 03-11B), BR 3262, BR 3299 e BR 3267, mais dois controles, testemunha e adubado com nitrogênio ( $50 \mathrm{~kg}$ de $\mathrm{N} \mathrm{ha}^{-1}$ ), em cada experimento, em blocos ao acaso. Para cada cultivar a resposta de cada estirpe inoculada variou dentro das características avaliadas. Porém, a estirpe BR 3302 mostrou uma constante em relação às características de biomassa e a produtividade para a cultivar Nova Era no experimento 1, com valores próximos ao tratamento adubado. Para as cultivares Pujante e Vinagre, nos experimentos 2 e 3, respectivamente, também foram observados resultados satisfatórios com a inoculação da estirpe BR 3302, com produtividade cerca de 22 e $11 \%$ superiores ao tratamento adubado com nitrogênio em cada experimento, porém, não diferindo das demais estirpes. Na média geral dos três experimentos independentes, foram observadas produtividades de grãos do feijão-caupi de 743 e $826 \mathrm{~kg} \mathrm{ha}^{-1}$ para os tratamentos com adubação nitrogenada e o tratamento inoculado com a estirpe BR 3302, respectivamente. Assim, a estirpe BR 3302 proporcionou os melhores resultados para as três cultivares de feijão-caupi.
\end{abstract}

Palavras-chave - Vigna unguiculata. Fixação biológica do nitrogênio. Biologia do solo.

\begin{abstract}
The interaction of cowpea with atmospheric N fixing bacteria can increase productivity and reduce production costs. In this study, it was evaluated in the field, the agronomic efficiency, through the accumulation of biomass of shoot, root, number and biomass of nodules, relative efficiency and productivity of three cultivars of cowpea (BRS New Era, BRS vibrant and Vinegar), in independent experiments, inoculated with five strains of rhizobia, BR 3302 (UFLA 3-84), BR 3301 (INPA 03-11B), BR 3262, BR 3299 and BR 3267, two controls, control and fertilized with nitrogen (50 kg of N ha-1) for each experiment, in blocks. For each cultivar the response of each strain inoculated varied within traits. However, strain BR 3302 showed a constant on the characteristics of biomass and productivity assessed, to cultivar Nova Era in Experiment 1, with values near the fertilized soil. For the cultivars Pujante and Vinagre, in experiments 2 and 3, respectively, were also observed satisfactory results with the inoculation of strain BR 3302, with productivity about 22 and $11 \%$ higher than the treatment fertilized with nitrogen in each experiment, however, did not differ on the other strains inoculated. The mean of three independent experiments, were observed grain yields of cowpea of 743 and $826 \mathrm{~kg} \mathrm{ha}^{-1}$ for the treatments with nitrogen and the treatment inoculated with strain BR 3302 , respectively. Thus, strain BR 3302 provided the best results for the three cultivars of cowpea.
\end{abstract}

Key Words - Vigna unguiculata. Biological nitrogen fixation. Soil biology.

\footnotetext{
* Autor para correspondência

${ }^{1}$ Recebido para publicação em 27/07/2009; aprovado em 12/10/2010

Pesquisa desenvolvida no curso de Pós-Graduação em Produção Vegetal da Universidade Federal do Tocantins, Campus Universitário de Gurupi, realizada com recursos do CNPq e CAPES PNPD

${ }^{2}$ Universidade Federal do Tocantins, Campus Universitário de Gurupi, Caixa Postal 66, Gurupi-TO, Brasil, 77.402-970, chagasjraf@uft.edu.br, gilrsan@uft.edu.br, rrfidelis@uft.edu.br, lillianfb@hotmail.com

${ }^{3}$ Programa de Pós-Graduação em Produção Vegetal, Universidade Federal do Tocantins, Campus Universitário de Gurupi, Caixa Postal 66, GurupiTO, Brasil, 77.402-970, wagnermicroxisto@yahoo.com.br
} 


\section{Introdução}

A cultura do feijão-caupi (Vigna unguiculata (L.) Walp.) é extremamente rústica, tolerante a altas temperaturas, à seca e com boas condições para adaptação e expansão das áreas atualmente exploradas. Apesar de ser considerada uma cultura de subsistência, assume expressiva importância sócio econômica no cenário da agricultura no Norte e Nordeste, constituindose na principal fonte de proteína de baixo custo para a alimentação humana, apresentando grande variabilidade entre as cultivares (BERTINE et al., 2009).

Historicamente, essa cultura apresenta baixa produtividade devido às condições de cultivos sem adoção de tecnologias avançadas (FREIRE et al., 2005). Isto porque, em condições de experimento e lavouras com melhor uso de tecnologia, o feijão-caupi tem apresentado alto potencial produtivo, o que em geral não tem sido explorado. Um dos fatores responsáveis pela baixa produtividade é a baixa fertilidade natural e dos teores de matéria orgânica dos solos, especialmente em áreas de cerrado.

A produtividade desta cultura poderia ser aumentada pelo uso de inoculantes de rizóbios eficientes, suprindo as necessidades de nitrogênio da planta (SILVA et al., 2006; ZILLI et al., 2009), baixando os custos de produção e elevando a renda do produtor. Em experimentos conduzidos em condições de campo, mostraram-se aumentos nos rendimentos de grãos em tratamentos inoculados com estirpes de rizóbio selecionados (MARTINS et al., 2003; ZILLI et al., 2006). Portanto, é imprescindível a difusão desta biotecnologia, de baixíssimo custo, para a cultura do feijão-caupi, considerando que a fixação biológica do nitrogênio é um processo ecológico e economicamente vantajoso que pode substituir os fertilizantes nitrogenados. No cerrado do Tocantins, entretanto, o uso de inoculantes na cultura do feijão-caupi ainda é muito limitado, necessitando de estudos de avaliação da fixação biológica do nitrogênio nesta cultura e da eficiência agronômica das estirpes de rizóbios nas condições de clima e solo do cerrado no Sul do Tocantins.

Assim, este trabalho teve por objetivo avaliar os efeitos da inoculação com estirpes de rizóbio em três cultivares de feijão-caupi, em condições de clima e solo do cerrado no Sul do Tocantins.

\section{Material e métodos}

No período de março a maio de 2009 foram conduzidos três experimentos de campo, inoculando- se sementes de feijão-caupi (cv. BRS Nova Era, BRS Pujante e Vinagre) com estirpes de rizóbio, sendo as cultivares avaliadas em experimentos independentes. Os experimentos foram implantados no Campo Experimental da Universidade Federal do Tocantins, no Campus Universitário de Gurupi/TO, localizado a $11^{\circ} 43^{\prime} 5 \mathrm{~S}$ e $49^{\circ} 04^{\prime} \mathrm{W}$ a $280 \mathrm{~m}$, conduzido de acordo com as recomendações da RELARE (Rede de Laboratórios para recomendação, padronização e difusão de tecnologia de inoculantes microbianos de interesse agrícola). O solo da área de cultivo apresentou as seguintes características: M.O: $22,1 \mathrm{~g} \mathrm{dm}^{-1}$; $\mathrm{pH}$ : 5,4; P: 7,3 mg dm${ }^{-3}$; K: 0,2 $\mathrm{cmol}_{\mathrm{c}} \mathrm{dm}^{-3}$; Ca: $4,2 \mathrm{cmol}_{\mathrm{c}} \mathrm{dm}^{-3}$; $\mathrm{Mg}$ : $1,71 \mathrm{cmol}_{\mathrm{c}} \mathrm{dm}^{-3}$; $\mathrm{Al}: 0,18 \mathrm{cmol}_{\mathrm{c}} \mathrm{dm}^{-3}$, na camada de $0-20 \mathrm{~cm}$ de profundidade.

Em cada experimento, o delineamento experimental utilizado foi em blocos casualizados com quatro repetições, com parcelas de $2 \mathrm{~m} \times 2 \mathrm{~m} \mathrm{e}$ espaçamento da cultura de $0,5 \mathrm{~m}$ entre linhas e 8-10 plantas por metro linear. Os tratamentos utilizados para cada cultivar de feijão-caupi foram inoculações com as estirpes BR 3301 (INPA 03-11B), BR 3302 (UFLA 3-84) (oriundas da coleção de culturas do laboratório de Microbiologia do Solo da Universidade Federal de Lavras), BR 3262, BR 3299 e BR 3267 (oriundas da coleção de culturas da Embrapa Agrobiologia), mais dois controles para cada experimento, sendo um com adubação nitrogenada (50 kg ha-1 no plantio) e uma testemunha (sem adubação nitrogenada e sem inoculação). Os inoculantes foram fornecidos em veículo turfoso e concentração mínima de rizóbio na ordem de $10^{9}$ células $\mathrm{g}^{-1}$ de inoculantes, sendo que a inoculação consistiu da aplicação de uma proporção de $500 \mathrm{~g}$ deste inoculante para cada $50 \mathrm{~kg}$ de sementes umedecidas em água potável.

As características avaliadas foram: massa seca da parte aérea (MSPA), da raiz (MSR), total (MST), número de nódulos ( $\mathrm{NN}$ ) e massa seca dos nódulos (MSN), aos 40 dias após o plantio, no início da floração, com a coleta de dez plantas na segunda linha de plantio em cada parcela. Ao final do ciclo, na colheita, a partir de 60 dias após a semeadura, foi avaliada a produtividade de grãos (após umidade corrigida para 13\%), em plantas competitivas da área útil de cada. Avaliou-se ainda a eficiência relativa (ER), calculada segundo a fórmula $\mathrm{ER}=(\mathrm{MSPA}$ inoculada/MSPA com N) x 100, em que MSPA inoculada é a matéria seca da parte aérea da planta com inoculação e MSPA com N é a matéria seca da parte aérea da planta com $\mathrm{N}$ mineral (LIMA et al., 2005). Os dados foram submetidos à análise de variância empregando-se o programa de análise estatística ASSISTAT, versão 7.4 beta, e as médias foram comparadas pelo teste de Duncan $(p<0,05)$. 


\section{Resultados e discussão}

Os resultados apresentados na Tabela 1 mostram, no experimento 1, com a cultivar Nova Era, que não ocorreram diferenças entre os tratamentos inoculados com as estirpes BR 3302, BR 3301, BR 3262 e o tratamento adubado com $\mathrm{N}$ (uréia) para MSPA e MST, sendo superiores $(\mathrm{p}<0,001)$ às estirpes BR 3299 e BR 3267. A estirpe BR 3262 foi a única que se mostrou superior quanto a MSR, em relação ao tratamento com adubação, apesar de não diferir significativamente das estirpes BR 3302, BR 3301 e BR 3267. Para o NN, os menores valores, entre os tratamentos inoculados, foram encontrados para o tratamento com a estirpe BR 3267, apesar de não diferir significativamente das estirpes BR 3301 e BR 3302. Para MSN as estirpes BR 3302, BR 3301 e BR 3262 foram superiores $(\mathrm{p}<0,001)$. Em relação à produtividade, foi observado que a estirpe BR 3302 proporcionaram rendimento superior aos outros tratamentos inoculados, porém com valores semelhantes ao tratamento adubado com ureia. As estirpes BR 3302, BR 3301 e BR 3262 contribuíram para a eficiência relativa (ER) superior em relação aos demais tratamentos, porém similar ao tratamento adubado (FIG. 1).

Tabela 1 - Biomassa, nodulação e produtividade de feijão-caupi, cultivares Nova Era, Pujante e Vinagre, inoculadas com estirpes de rizóbio. Experimentos 1, 2 e $3^{(1)}$

\begin{tabular}{|c|c|c|c|c|c|c|}
\hline Isolados/ estirpes & MSPA (g) & $\operatorname{MSR}(g)$ & $\operatorname{MST}(\mathrm{g})$ & $\mathrm{NN}^{(2)}$ & $\mathrm{MSN}(\mathrm{mg})^{(2)}$ & Prod. ${ }^{(4)} \mathrm{kg} \mathrm{ha}^{-1}$ \\
\hline \multicolumn{7}{|c|}{ Experimento 1: cv Nova Era } \\
\hline BR 3302 (UFLA 3-84) & $14,09 \mathrm{a}$ & $1,27 \mathrm{ab}$ & $14,36 \mathrm{a}$ & $27 \mathrm{ab}$ & $427 \mathrm{ab}$ & $680 \mathrm{a}$ \\
\hline BR 3301 (INPA 3-11B) & $13,02 \mathrm{ab}$ & $0,99 \mathrm{ab}$ & $14,01 \mathrm{a}$ & $25 \mathrm{ab}$ & $420 \mathrm{ab}$ & $590 \mathrm{c}$ \\
\hline BR 3262 & $14,22 \mathrm{a}$ & $1,38 \mathrm{a}$ & $15,60 \mathrm{a}$ & $33 \mathrm{a}$ & $483 \mathrm{a}$ & $513 \mathrm{~d}$ \\
\hline BR 3299 & $8,25 \mathrm{c}$ & $0,98 \mathrm{bc}$ & $9,23 \mathrm{c}$ & $36 a$ & $108 \mathrm{~d}$ & $520 \mathrm{~d}$ \\
\hline BR 3267 & $9,91 \mathrm{bc}$ & $1,33 \mathrm{ab}$ & $11,24 \mathrm{bc}$ & $18 \mathrm{~b}$ & $400 \mathrm{~b}$ & $647 \mathrm{bc}$ \\
\hline Testemunha & $8,29 \mathrm{c}$ & $0,69 \mathrm{~d}$ & $8,98 \mathrm{c}$ & $2 \mathrm{c}$ & $7,3 \mathrm{e}$ & $653 \mathrm{bc}$ \\
\hline Adubado & $14,06 \mathrm{a}$ & $0,95 \mathrm{bc}$ & $15,01 \mathrm{a}$ & $1 \mathrm{c}$ & $0,5 \mathrm{e}$ & $693 \mathrm{a}$ \\
\hline $\mathrm{CV}(\%)^{(3)}$ & 17,14 & 22,99 & 16,05 & 34,93 & 15,74 & 7,43 \\
\hline \multicolumn{7}{|c|}{ Experimento 2: cv Pujante } \\
\hline BR 3302 (UFLA 3-84) & $19,53 \mathrm{a}$ & $0,72 \mathrm{ab}$ & $20,25 \mathrm{a}$ & $27 \mathrm{ab}$ & $425 \mathrm{a}$ & $888 \mathrm{a}$ \\
\hline BR 3301 (INPA 3-11B) & $14,74 \mathrm{~b}$ & $0,81 \mathrm{ab}$ & $15,65 \mathrm{bc}$ & $34 \mathrm{a}$ & $466 \mathrm{a}$ & $848 \mathrm{ab}$ \\
\hline BR 3262 & $11,41 \mathrm{c}$ & $0,64 \mathrm{bc}$ & $12,05 \mathrm{de}$ & $30 \mathrm{ab}$ & $181 \mathrm{~b}$ & $815 \mathrm{ab}$ \\
\hline BR 3299 & $8,49 \mathrm{~d}$ & $0,74 \mathrm{ab}$ & $9,23 \mathrm{f}$ & $34 \mathrm{a}$ & $492 \mathrm{a}$ & $753 \mathrm{~cd}$ \\
\hline BR 3267 & $11,36 \mathrm{c}$ & $0,65 \mathrm{bc}$ & $12,01 \mathrm{de}$ & $24 \mathrm{ab}$ & $423 \mathrm{a}$ & $783 \mathrm{bc}$ \\
\hline Testemunha & $15,74 \mathrm{~b}$ & $0,71 \mathrm{ab}$ & $16,45 \mathrm{~b}$ & $3 \mathrm{c}$ & $8,8 \mathrm{c}$ & $646 \mathrm{e}$ \\
\hline Adubado & $16,08 \mathrm{~b}$ & $0,87 \mathrm{ab}$ & $16,95 \mathrm{~b}$ & $1 \mathrm{c}$ & $7,3 \mathrm{c}$ & $723 \mathrm{~cd}$ \\
\hline CV $(\%)$ & 9,52 & 20,94 & 9,42 & 31,96 & 35,01 & 6,38 \\
\hline \multicolumn{7}{|c|}{ Experimento 3: cv Vinagre } \\
\hline BR 3302 (UFLA 3-84) & $15,68 \mathrm{a}$ & $0,91 \mathrm{a}$ & $16,59 \mathrm{a}$ & $33 \mathrm{a}$ & $428 \mathrm{~b}$ & $910 \mathrm{a}$ \\
\hline BR 3301 (INPA 3-11B) & $12,85 \mathrm{~b}$ & $0,54 \mathrm{bc}$ & $13,39 \mathrm{~b}$ & $36 a$ & $475 \mathrm{ab}$ & $838 \mathrm{ab}$ \\
\hline BR 3262 & $15,93 \mathrm{a}$ & $0,63 \mathrm{bc}$ & $16,56 \mathrm{a}$ & $18 \mathrm{~b}$ & $554 \mathrm{a}$ & $855 \mathrm{ab}$ \\
\hline BR 3299 & $10,90 \mathrm{bc}$ & $0,75 \mathrm{ab}$ & $11,65 \mathrm{bc}$ & $26 \mathrm{ab}$ & $457 \mathrm{ab}$ & $905 \mathrm{a}$ \\
\hline BR 3267 & $9,88 \mathrm{c}$ & $0,64 \mathrm{bc}$ & $10,52 \mathrm{~cd}$ & $21 \mathrm{~b}$ & $507 \mathrm{ab}$ & $865 \mathrm{ab}$ \\
\hline Testemunha & $10,95 \mathrm{bc}$ & $0,81 \mathrm{ab}$ & $11,76 \mathrm{bc}$ & $4 \mathrm{c}$ & $49 \mathrm{~d}$ & $763 \mathrm{~d}$ \\
\hline Adubado & $11,49 \mathrm{bc}$ & $0,61 \mathrm{bc}$ & $12,10 \mathrm{bc}$ & $2 \mathrm{c}$ & $7,3 \mathrm{~d}$ & $813 \mathrm{c}$ \\
\hline CV (\%) & 11,65 & 25,31 & 11,15 & 32,30 & 25,2 & 6,42 \\
\hline
\end{tabular}

${ }^{(1)}$ Médias seguidas de mesma letra, nas colunas, não diferem entre si pelo teste Duncan a $5 \%$. ${ }^{(2)}$ Dados transformados por $\mathrm{X}+0,5$. ${ }^{(3)}$ Coeficiente de Variação. ${ }^{(4)}$ Prod.= Produtividade 
No experimento 2 com a cultivar Pujante, a estirpe BR 3302 foi superior $(p<0,001)$ aos outros tratamentos para MSPA e MST. Quanto a MSR, as estirpes BR 3262 e BR 3267 apresentaram valores inferiores aos outros tratamentos. Para o NN todas as estirpes apresentaram nodulação superior aos tratamentos adubado e testemunha. Para a MSN o menor valor, entre as estirpes inoculadas, foi encontrado para a estirpe BR 3262. Em relação à produtividade, as estirpes BR 3302, BR 3301 e BR 3262 apresentaram mais de $800 \mathrm{~kg}$ por hectare, sendo significativamente superiores ao tratamento adubado (TAB. 1). Para a ER, destaque, também, para a estirpe BR 3302, com valores superiores aos outros tratamentos (FIG. 1).

No experimento 3, com a variedade Vinagre, foram observados valores superiores $(\mathrm{p}<0,001)$ quanto a MSPA e MST para as estirpes BR 3302 e BR 3262. Para a MSR destaque para as estirpes BR 3302 e BR 3299. Em relação ao NN as estirpes BR 3302, BR 3301 e BR 3299 foram superiores $(p<0,01)$. Já para MSN destaque para a estirpe BR 3262, porém não diferindo das demais estirpes inoculadas. Todas as estirpes proporcionaram produtividades significativamente superiores aos tratamentos testemunha e adubado (TAB. 1). Para a ER os maiores valores foram para as estirpes BR 3302 e 3262 (FIG. 1).

O feijão-caupi é capaz de nodular com diversas espécies de bactérias do grupo rizóbio, especialmente do gênero Bradyrhizobium, Rhizobium e Sinorhizobium (RUMJANEK et al., 2005; ZILLI et al., 2006). Esta característica, apesar de representar uma vantagem ecológica para a adaptação deste vegetal, é um fator limitante ao uso de inoculantes em sistemas agrícolas (XAVIER et al., 2006). Isto porque normalmente a cultura apresenta baixa especificidade de nodulação.

Desta forma, apesar de ser uma das leguminosas com maior capacidade em fixar nitrogênio atmosférico nos sistemas agrícolas, a ocorrência de nodulação espontânea e, principalmente, a falta de resultados positivos em condições de campo, faz com que a prática de inoculação ainda não seja amplamente usada para esta cultura no Brasil (FREIRE FILHO et al., 2005).

A estirpe BR 3302 mostrou-se constante na relação MSPA, MSR, MST, NN, MSN, ER e produtividade, para a cultiva Nova Era no experimento 1 (TAB. 1), com valores próximo ao tratamento adubado, tornando visível a resposta positiva desta cultivar a esta estirpe.

Para as cultivares Pujante e Vinagre, nos experimentos 2 e 3, respectivamente, também foram observados resultados satisfatórios com a inoculação da estirpe BR 3302, com produtividade cerca de 22 e $11 \%$ superiores ao tratamento adubado de cada experimento.
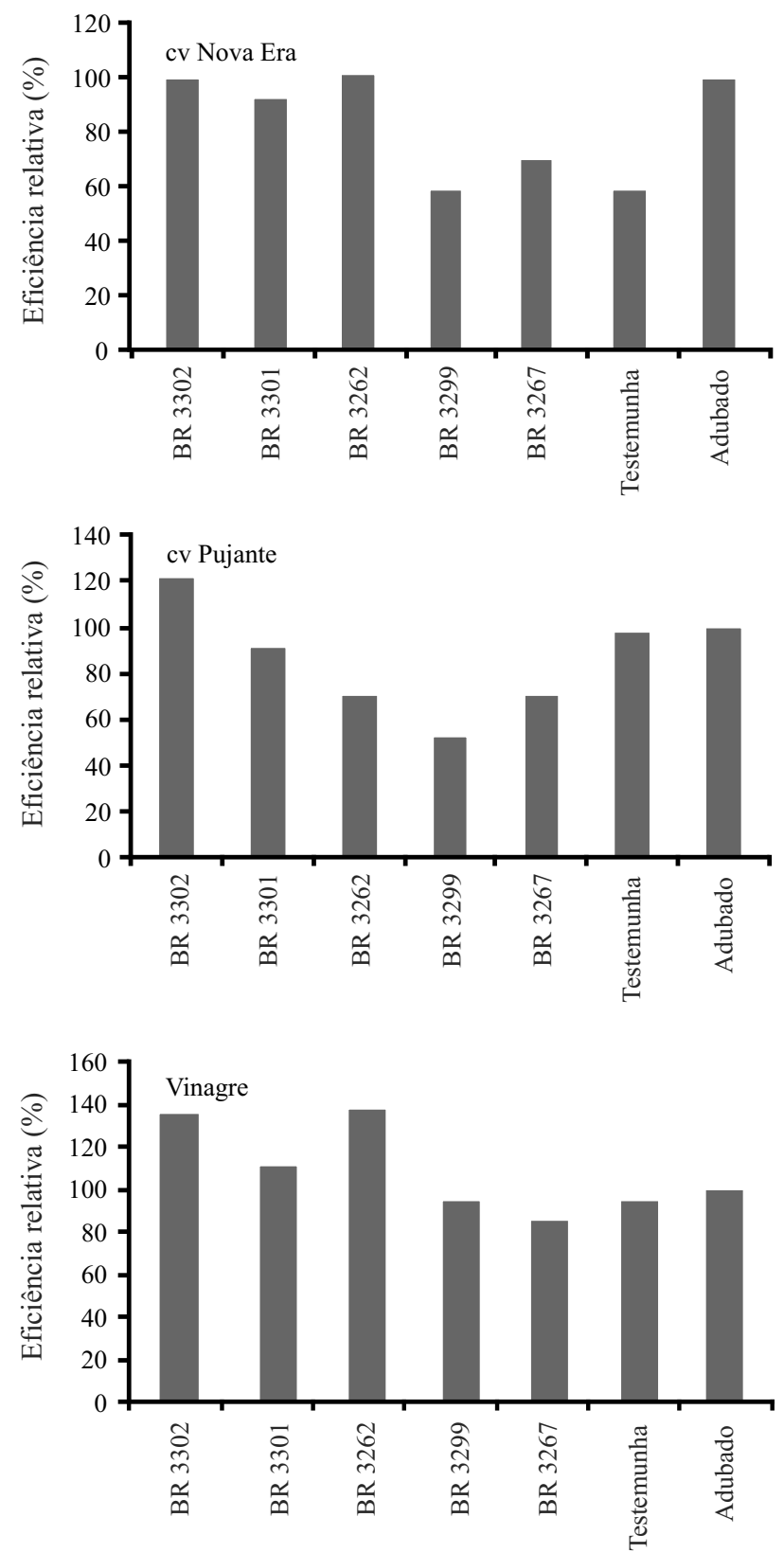

Figura 1 - Eficiência Relativa de feijão-caupi, cultivares Nova Era, Pujante e Vinagre, dos tratamentos inoculados com estirpes de rizóbios e testemunha, em relação ao tratamento adubado com nitrogênio (uréia). Experimentos 1, 2 e 3

Algumas estirpes, entre elas BR 3302, BR 3301, BR 3267 e BR 3262, foram testadas em outros trabalhos (ZILLI et al., 2006) e mostraram bom desempenho, tanto de eficiência quanto competitividade.

Analisando os dados conjuntamente, observouse que a média geral de produtividade diferiu de forma significativa entre os diferentes experimentos, ocorrendo maior produtividade nas cultivares Pujante e Vinagre 
inoculadas com a estirpe BR 3302. Na média geral dos três experimentos, foram observadas produtividades de grãos de feijão-caupi de 743 e $826 \mathrm{~kg} \mathrm{ha}^{-1}$ para os tratamentos com adubação nitrogenada e para o tratamento inoculado com a estirpe BR 3302, respectivamente, sendo as médias obtidas nesses tratamentos significativamente superiores ao controle de cada experimento (TAB. 1). Porém, as médias de produtividades do feijão-caupi inoculado foram inferiores aos encontrados em trabalhos de campo em outras regiões (ZILLI et al., 2006; GUALTER et al., 2007). Mas, os experimentos em outras regiões receberam adubação de plantio com fósforo, potássio e micronutrientes, o que não ocorreu neste experimento, visando à realidade dos produtores na região de cerrado no Sul do Tocantins.

Os resultados encontrados no presente trabalho corroboram aos encontrados por Nascimento et al. (2008, 2010) e Zilli et al. (2009), avaliando o desenvolvimento e a produtividade do feijão-caupi em condições de campo, onde a inoculação com estirpes recomendadas permitiram produção de biomassa e grãos no feijão-caupi semelhantes à da adubação com $50 \mathrm{~kg} \mathrm{ha}^{-1}$ de $\mathrm{N}$.

As estirpes testadas apresentaram grande eficiência na fixação do nitrogênio, parte delas com produção de biomassa equivalente a $90 \%$, ou mais, da biomassa produzida pelo tratamento com $\mathrm{N}$ mineral (TAB. 1). Comportamento semelhante com relação à biomassa do feijão-caupi inoculado com estirpes referência foi encontrado por Soares et al. (2006), Melo et al. (2009), Zilli et al. (2009) e Nascimento et al. (2010), tanto em condições de casa de vegetação como de campo.

À medida que houve aumento da biomassa seca de nódulos, aumentou também a matéria seca da parte aérea no experimento 1 com a cultivar Nova Era (TAB. 1), sendo encontrado um alto coeficiente de correlação $(\mathrm{r}=0,84)$ entre as duas variáveis. Porém, não foi observada correlação entre estas variáveis nos experimentos 2 (cv. Pujante) e 3 (cv. Vinagre). Pimratch et al. (2004) sugeriram que o acúmulo de MSPA é a característica mais confiável para a seleção de cultivares com maior potencial simbiótico em solos com baixa disponibilidade de nitrogênio. O potencial em fixar nitrogênio das estirpes recomendadas foi de fundamental importância para a produção de biomassa, o que pode estar relacionado com a efetiva capacidade de fornecimento de nitrogênio pelas estirpes testadas, demonstrado pela correlação positiva encontrada enre a eficiência relativa e a produção de massa seca da parte aérea para as cultivares Nova Era $(\mathrm{r}=0,78)$, Pujante $(r=0,89)$ e Vinagre $(r=0,88)$, em seus respectivos experimentos. Resultados semelhantes foram relatados por Silva (2007), o qual também encontrou alta correlação envolvendo as variáveis de biomassa relativa de plantas de amendoim inoculadas com isolados de
Bradyrhizobium spp., cultivadas em solo da Zona da Mata de Pernambuco.

De acordo com os dados apresentados, respostas positivas de inoculação serão mais facilmente obtidas em áreas com baixa população de rizóbio nodulantes de feijão-caupi estabelecida no solo, fato que ocorreu onde foram implantados os experimentos, tendo o tratamento controle uma baixa nodulação.

Considerando-se que os solos onde foram implantados os experimentos apresentavam limitações quanto à fertilidade, a produtividade da cultura de feijão-caupi obtida com inoculação foi expressiva em relação ao tratamento controle, mostrando a possibilidade de aumento da produtividade de grãos de feijão-caupi por parte dos agricultores com a adoção da tecnologia de inoculação. Porém, existe necessidade de experimentos de inoculação com adubação de plantio, visando aumento da produtividade.

\section{Conclusão}

A estirpe BR 3302 (UFLA 3-84) proporciona melhores resultados para as três cultivares de feijãocaupi, nos três experimentos, semelhantes ao tratamento adubado com nitrogênio. Porém, ainda é preciso a realização de outros experimentos para avaliar melhor seu potencial.

\section{Agradecimentos}

A CAPES-PNPD e CNPq-Universal.

\section{Referências}

BERTINI, C. H. C. M.; TEÓFILO, E. M.; DIAS, F. T. C. Divergência genética entre acessos de feijão caupi do banco de germoplasma da UFC. Revista Ciência Agronômica, v. 40, n. 01, p. 99-105, 2009.

FREIRE FILHO, F. R.; LIMA, J. A; RIBEIRA, A. Feijão caupi: avanços tecnológicos. Brasília: Embrapa Informações Tecnológicas, 2005. 519 p.

GUALTER, R.M.R. et al. Avaliação dos efeitos da inoculação de feijão-caupi (Vigna unguiculata [L.] Walp.) com Bradyrhizobium elkanii. Revista Brasileira de Agroecologia, v. 02, n. 02, p. 637-640, 2007.

LIMA, A. S.; PEREIRA, J. P. A. R.; MOREIRA, F. M. S. Diversidade fenotípica e eficiência simbiótica de estirpes de Bradyrhizobium spp. de solos da Amazônia. Pesquisa Agropecuária Brasileira, v. 40, p. 1095-1104, 2005. 
MARTINS, L. M. et al. Contribution of biological nitrogen fixation to cowpea: a strategy fot improving grain yield in the semi-arid region of Brazil. Biology and Fertility of Soil, v. 38, p. 333-339, 2003.

MELO, S. R.; ZILLI, J. E. Fixação biológica de nitrogênio em cultivares de feijão-caupi recomendadas para o Estado de Roraima. Pesquisa Agropecuária Brasileira, v. 44, n. 09, p. 1177-1183, 2009.

NASCIMENTO, L. R. S. et al. Nodulação e produção do caupi (Vigna unguiculata L.) sob efeito de plantas de cobertura e inoculação. Revista Brasileira de Ciência do Solo, v. 32, p. 579-587, 2008.

NASCIMENTO, L. R. S. et al. Eficiência de isolados de rizóbios natives do agreste paraibano em caupi. Revista Brasileira de Ciências Agrárias, v. 05, n. 01, p. 36-42, 2010.

PIMRATCH, S. et al. Evolution of seven peanut genotypes for nitrogen fixation and agronomic traits. Songklanakarin Journal Science Technology, v. 26, n. 03, p. 295-304, 2004.

RUMJANEK, N. G. et al. Fixação biológica de nitrogênio. In: FREIRE FILHO, F. R. et al. Feijão Caupi: avanços tecnológicos. Brasília: Embrapa Informações Tecnológicas, 2005. p. 281-335.
SILVA, M. F. Efetividade da inoculação com Bradyrhizobium spp. Em amendoim cultivado em solo da zona da mata de Pernambuco. 2007. 56 f. Dissertação (Mestrado em Ciência do Solo). Universidade Federal Rural de Pernambuco, Recife.

SILVA, V. N.; SILVA, L. E. S. F.; FIGUEIREDO, V. B. Atuação de rizóbio com rizobactéria promotora de crescimento em plantas na cultura do caupi (Vigna unguiculata [L.] Walp.). Acta Scientiarum Agronomy, v. 28, n. 03, p. 407-412, 2006.

SOARES, A. L. L. et al. Eficiência agronômica de rizóbios selecionados e diversidade de populações nativas nodulíferas em Perdões (MG): II - feijoeiro. Revista Brasileira de Ciência do Solo, v. 30, p. 803-811, 2006.

XAVIER, G. R. et al. Especificidade simbiótica entre rizóbio e acessos de feijão-caupi de diferentes nacionalidades. Revista Caatinga, v. 19, n. 01, p. 25-33, 2006.

ZILLI, J. E. et al. Caracterização e avaliação da eficiência simbiótica de estirpes de Bradyrhizobium em caupi nos solos de cerrados. Pesquisa Agropecuária Brasileira, v. 41, p. 811-818, 2006.

ZILLI, J. E. et al. Contribuição de estirpes de rizóbio para o desenvolvimento e produtividade de grãos de feijão-caupi em Roraima. Acta Amazonica, v. 39, n. 04, p. 749-758, 2009. 